\title{
Urethroplasty with dorsal buccal mucosa graft. Is it still the method of choice in long term urethral stenosis?
}

\author{
Carlo Pavone, Dario Fontana, Ninfa Giacalone, Nino Dispensa, Marco Vella, Alchiede Simonato \\ UOC Urologia, Dipartimento di Discipline Chirurgiche, Oncologiche e Stomatologiche, Università degli studi di Palermo, Italy.
}

\begin{abstract}
Summary The aim of our work was to evaluate the long-term changes in symptoms (median 42 months) and to analyze data for any negative predictive factors for the application of the procedure, in patients who underwent to urethroplasty with dorsal buccal mucosa graft. During the period from 2010 to 201527 patients were examined. Than they underwent urethroplasty using dorsal buccal mucosa graft (graft of $4 \times 2.5 \mathrm{~cm}$ ). The evaluation of symptoms has been addressed through the application of the IPSS Quality of Life Questionnaire (International Prostatic Symptoms Score) and the evaluation of urinary flow has been carried out by a comparative analysis between the pre- and post-operative uroflowmetry. As our study has shown, data obtained by the screening tests in the post-operative follow-up indicate that there is an increase in the maximum flow of urine until 1 month after surgery. The results in the long-term follow-up are different because they show a partial reduction of the maximum flow although it is maintained around an average value of $23 \mathrm{ml} / \mathrm{s}$ being still higher than the maximum flow in the pre-operative period. According to our results it follows that there is a low failure rate of the procedure after a median of 42 months. Only in patients with urethral stenosis longer than $2 \mathrm{~cm}$, a lower long term success is achieved. From what we could observe, this length of the stenosis seems to be the only negative predictive factor for long-term maintenance of a good Quality of Life in patients undergoing the procedure. The results obtained from our study confirm literature data according to which, the gold standard for $2-\mathrm{cm}$ long bulbar urethral stricture whose lumen is well preserved with circumferential spongiofibrosis limited to 1-2 $\mathrm{mm}$ is the dorsal graft urethroplasty with buccal mucosa that in our study showed success rates higher of $80 \%$ after a median follow up of 42 months and a percentage of relapse-free patients of $82.1 \%$ ( median 3.5 years).
\end{abstract}

KEY WORDS: Urethroplasty; Urethral stenosis; Urology; Andrology.

Submitted 22 June 2016; Accepted 7 September 2016

\section{INTRODUCTION}

The urethral stenosis is a scar of sub-epithelial tissue of the corpus spongiosum which narrows the urethral lumen. The incidence is approximately 1 case in 10,000 in men aged between 25 and 65 years, and 1 case in 1000 in men over 65 years of age. The process leading to urethral stenosis provides for an initial phase in which prevails the inflammatory or hemorrhagic component and a later stage characterized by sclerosis and fibrosis. The different anatomical structure of the urethra (anterior and posterior urethra) also explains the different causes of urethral stenosis between the two segments.

Today, the most common forms of stenosis are those involving bulbar urethra. In this tract, idiopathic stenosis tends to occur both in adolescents and in young adults, whereas iatrogenic stenosis could occur at any age due to catheterization, mainly in bulbar urethra junction and penis, or due to transurethral resection of the prostate (TURP) at the proximal part of bulbar urethra. The pathogenesis has not yet been fully elucidated, histopathological studies of urethral stenosis have shown how the main change at the base of this process is the metaplasia of urethral epithelial which is the transition from a pseudostratified columnar epithelium to a stratified squamous epithelium (2).

This change leads to an increased epithelial fragility especially during the distension caused by urination and so causes an extravasation of urine. This will lead to subepithelial fibrosis $(3,4)$. The formation and fusion of microscopic foci of fibrosis will lead in the following years the formation of macroscopic plaques, which will form a ring restricting totally the urethral lumen. The latest studies carried out the molecular level indicate that in the urethra there is a disproportionate deposition of type 1 collagen compared to type 3 collagen, which instead predominates in other fibrotic processes (5).

This characteristic is more pronounced in the urethral stenosis due to traumatic than in non-traumatic stenosis (6), in which there is often a surprisingly high content of smooth muscle (4). It also appears that this spongiofibrosis process may be induced by the neuronal nitric oxide synthasel or by the over-expression of a growth factor of the connective tissue, identified as the cause of other fibrotic diseases (7). The urethral stenosis left untreated is a highly pathogenic substrate, as a result of chronic and recurrent infections or the establishment of high pressures during emptying the bladder, with the complications that may first concern the lower urinary tract and then the upper one. At lower urinary level, the organ more affected is definitely the bladder in which it could be observed a wall thickening, with formation of trabeculae and following sacculation of the bladder as well as to an incomplete emptying of the organ and to a possible occurrence of lithiasis. At upper urinary level a 
hydroureteronephrosis process might occur developing renal failure (8).

Diagnostics plays a key role in the staging of urethral stricture disease, and in the planning of the most efficient therapeutic strategy. The clinical and radiological examinations used in the stenosis study are: urine culture, uroflometry, retrograde urethrogram, voiding cystourethrography, ultrasonography and urethroscopy.

There is a large number of possible options for surgical treatment proposed over the time. The choices in the treatment of anterior urethral stenosis, depending on the place and the length of the stenosis, as well as by history of any previous surgeries, are: urethral dilation, endoscopic urethrotomy according to Sachse or by the use of the laser, and the various techniques of reconstructive urethroplasty.

\section{MATERIALS AND METHODS}

From 2010 to 2015, 27 patients underwent urethroplasty surgery using dorsal buccal mucosa graft (graft of $4 \mathrm{x}$ $2.5 \mathrm{~cm}$ ). After transurethral injection of methylene blue, the procedure involves a longitudinal skin incision on the perineal floor with blunt cutaneous dissection and section of Colles fascia that allows the identification of bulbospongiosus muscles and their bilateral dissection.

The corpus spongiosum of the urethra is so exposed making possible the Isolation of the bulbar urethra and its subsequent lateralization. The dorsal urethral surface is rotated of $180^{\circ}$. This maneuver allows to incise the stenotic segment longitudinally throughout its length. The withdrawal of the buccal mucosa could be performed both before and/or after the perineal incision, and the taken patch is positioned and sutured at the level of the corpora cavernosa.

The aim of our work was to evaluate longterm changes in symptoms (average 42 months) and to analyze data for any negative predictive factor for the success of the procedure in patients undergoing urethroplasty with dorsal buccal mucosa graft. The age of the analyzed sample was 43.2 years (range 2477). Among the target population 3 patients (8\%) have multiple stenosis, 1 patient $(2 \%)$ an anterior penile stenosis, 4 patients (9.8\%) membranous stenosis, and the remaining 19 patients (80.2\%) a bulbar stenosis.

The evaluation of symptoms was addressed by the application of the IPSS Quality of Life Questionnaire (International Prostatic Symptoms Score), to highlight significant variation between pre-operative and post- operative period whereas for the evaluation of the urinary flow a comparative analysis was carried out between the pre- and post-operative uroflowmetry values with particular attention to the maximum flow rate $\left(Q_{\max }\right)$ that showed a significant improvement, as it is explained below.

\section{RESULTS}

The obtained results from our work confirm literature data according to which, the gold standard for 2-cm long bulbar urethral stenosis in which lumen is well preserved and the circumferential spongiofibrosis is limited to 1-2 $\mathrm{mm}$, is the dorsal graft urethroplasty with buccal mucosa, that showed in our study a high success rate of $80 \%$ after a median follow up of 42 months and a percentage of relapse-free patients of $82.1 \%$ (median 3.5 years). As mentioned above, in our series replies to the IPSS questionnaire related to quality-of-life before surgery showed an average score of 5.86, whereas in the post-operative period the average score was 3.4 during the 5-year follow-up period (Figure 1).

We particularly paid attention to values of maximum flow, which allowed us to see an improvement from an average of $7.4 \mathrm{ml} / \mathrm{s}$ (range $5-11 \mathrm{ml} / \mathrm{s}$ ) to reach a mean value of $23 \mathrm{ml} / \mathrm{s}$ (range $5-42 \mathrm{ml} / \mathrm{s}$ ) one month after surgery and of $21.16 \mathrm{ml} / \mathrm{s}$ in the fifth year following the procedure (Figure 2).

The ultrasound of corpus spongiosum did not demonstrate a significant change between the pre- and postoperative period.

Figure 1.

IPSS score related to the value of $Q_{\max }$ during the preoperative period, 1 month after surgery and at long term (median of 3.5 years).

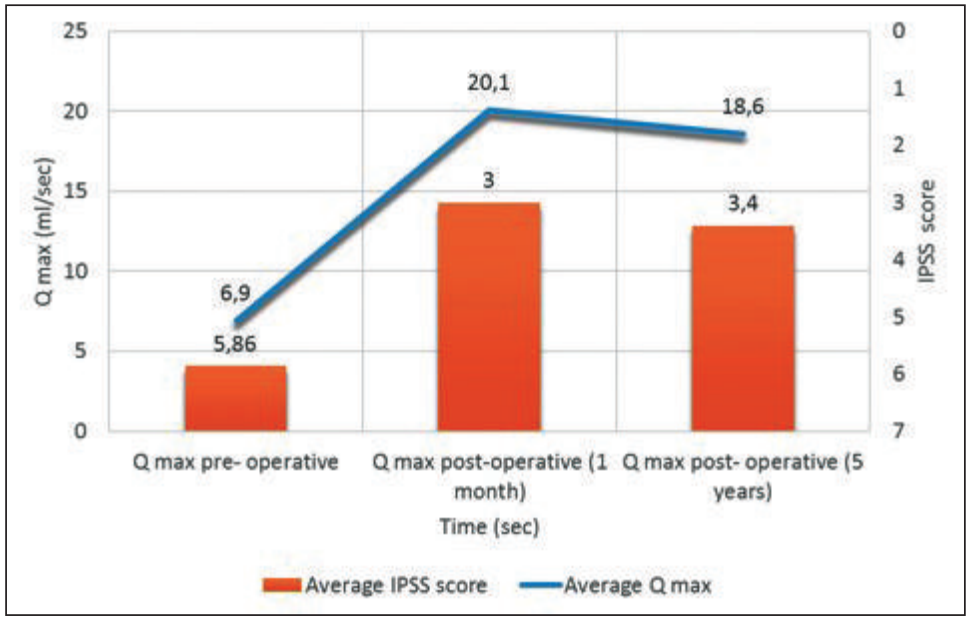

Figure 2.

Maximum pre-operative flow rate $\left(Q_{\max }\right)$, one month after surgery, and long-term (median of 3.5 years).

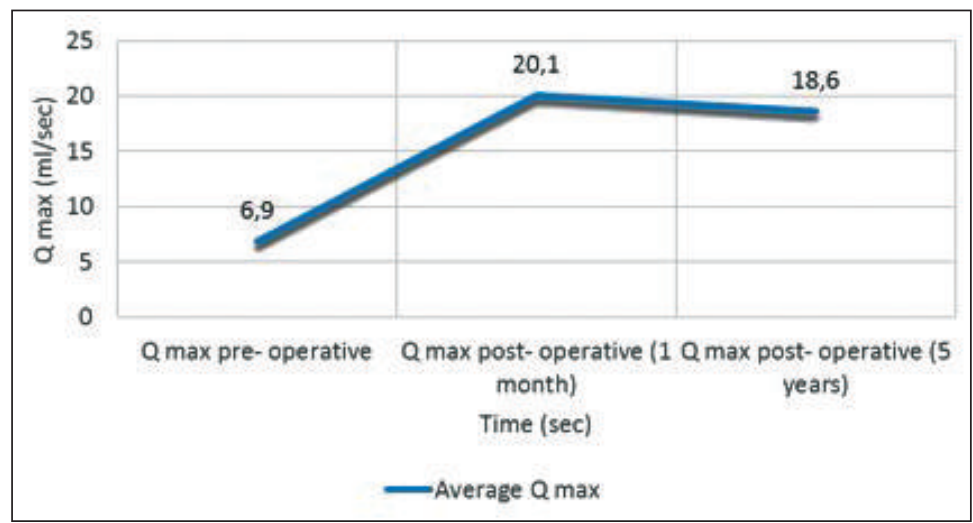




\section{Conclusions}

Taking into consideration the many and efficient treatment methods for urethral stenosis and the results obtained, we could state that at long-term follow-up of patients with urethral stenosis, the most successful technique is the urethroplasty with buccal mucosa graft.

As our study has shown, the results of the examinations in the follow-up after surgery indicate that, as described above, there is an increase in the maximum flow of urine until one month after surgery.

In the long-term follow-up we observed a partial reduction of the maximum flow, although the maximum flow was maintained at an average value of $23 \mathrm{ml} / \mathrm{s}$, which was still higher than the maximum flow in the pre-operative period. According to our results, a low failure rate of the procedure was shown after an average of 42 months. Only patients with urethral stenosis longer than $2 \mathrm{~cm}$ were slightly less successful. From what we could observe, only the length of the stenosis seemed to be the only negative predictive factor for long-term maintenance of good quality of life in patients undergoing this procedure.

\section{References}

1. Mundy AR. The treatment of sphincterstrictures. Br J Urol. 1989; 64:626-628

2. Chambers RM, Baitera B. The anatomy of the uretra stricture. $\mathrm{Br}$ J Urol. 1977; 49:545-551.

3. Meria P, Anidjar M, Brouland JP, et al. An experimental model of bulbar uretra stricture in rabbits using endoscopic rediofrequency coagulation. Urology. 1999; 53:1054-1057.

4. Singh M, Blandy JP. The pathology of uretra stricture. J Urol. 1976; 115:673-676.

5. Baskin LS, Constantinescu SC, Howard PS, et al. Biochemical characterization and quantification of the collagenous components of urethral stricture tissue. J Urol. 1993; 150:642-647.

6. Cavalcanti AG, Costa WS, Baskin LS, et al. A morphometric analysis of bulbar uretra Strictures. BJU Int. 2007; 100:397-402.

7. Zhang P, Shi M, Wei Q, et al. Increased expression of connective tissue grow factor in patients with uretra stricture. Tohoku J Exp Med, 2008; 215:199-206.

8. Romero Perez P, Mira Llinaries A. Complications of the lower urinary tract secondary to uretra stenosis. Actas Urol Esp. 1996; 20:786-793

\section{Correspondence}

Carlo Pavone, MD

Dario Fontana, MD

dariofontana1987@yahoo.it

Ninfa Giacalone, MD

Nino Dispensa, MD

Marco Vella, MD

Alchiede Simonato, MD

UOC Urologia, AOUP P. Giaccone

Via del Vespro 127 - 90100 Palermo, Italy 